\title{
Einige Erfahrungen bei der Behandlung der Hämoptyse.
}

\author{
Von
}

N. Lunde, dirigierender Arzt.

Bei Patienten, die an Lungentuberkulose leiden, ist die Hämoptyse diejenige Komplikation, welche den häufigsten und stärksten Schrecken bei dem Patienten und seiner Umgebung verursacht. Sie erfordert immer eine mehr oder weniger eingreifende Behandlung und ist gewöhnlich ein dankbares Objekt für eine solche. Wohl kann - cum grano salis - gesagt werden, dass die Blutung gewöhnlich von selbst aufhört, und zwar wesentlich auf Grund der von den Geweben aufgesaugten Thrombokinase. Aber es muss hier immer daran erinnert werden, dass es für den Patienten in keinem Falle ohne Bedeutung ist, in welch hohem Grade und in wie langer Zeit er den durch die Hämoptyse hervorgerufenen und sie begleitenden Zuständen ausgesetzt ist. Man sieht auch häufig genug $\mathrm{F}$ älle, in welchen mit Unterbrechung das Blut in Tagen, Wochen bis Monaten sickert. Bisweilen treten auch mit kürzeren oder längeren Intervallen bedeutende Blutungen auf, die in verschiedener Weise das Leben des Patienten in Gefahr bringen.

Die Hämoptyse tritt sehr häufig nach der einen oder anderen Gelegenheitsursache auf, gewöhnlich Überanstrengung der einen oder anderen Art und kündigt sich gerne durch Fieber an (Allgemeinund Herdreaktion einer zu grossen Dose Autotuberkulin). Sie tritt auch nicht selten auf in direktem Anschluss an eine Ursache von mehr oder weniger traumatischer Natur - ein Fall, Stoss, ungezügelte Bewegungen; unbeherrschtes Lachen oder Husten, Hinaufspringen der Treppen usw. Sie muss hier .wohl gewöhnlich hervorgerufen sein durch das Bersten eines grösserén oder kleineren Gefässes oder durch den Abstoss einer erweichten Masse in einer grösseren oder kleineren Kaverne. Zuweilen kann sie auch wie der Blitz aus heiterem Himmel kommen ohne eine eigentlich nachweislich veranlassende Ursache. Es muss daher angenommen werden, dass hier entweder doch ein 
reaktionserregendes Moment vorliegt, oder dass die Krankheit sich in einer progredienten Periode mit Erweichung des einen oder anderen Herdes befindet. Als begünstigende Momente für das Entstehen der Hämoptyse hat man hohen Blutdruck genannt, vermehrtes Flüssigkeitsvolumen im Organismus, unter anderem hervorgerufen durch reichliche Zufuhr von Fluida oder durch grosse relative Feuchtigkeit in der umgebenden Luft. Auch niederen Luftdruck hat man in dieser Verbindung genannt, aber dies dürfte doch eine sehr kleine Rolle spielen, wenn die Luftdruckveränderung nicht schnell geschieht, und nicht andere Faktoren hinzutreten - wie z. B. starke Anstrengungen mit forcierten schnellen und tiefen Atemzügen. Die Gefahren bei einer Hämoptyse können wesentlich in folgende Möglichkeiten zusammengefasst werden: Aspiration eines grösseren orler kleineren Teiles des Blutes mit darauf folgender Pneumonie oder Ausbreitung des tuberkulösen Prozesses; direkte Verblutung usque ad mortem ist selter; dagegen ist bei Aspiration von grösseren Blutmengen die Gefahr des Erstickungstodes bei weitem grösser, hauptsächlich durch ungenügende Expektoration. (Liegt die Gefahr des Erstickens vor, oder hat die Respiration sogar schon aufgehört, muss man zu einer manuellen Entfernung des Blutes und der Koagula in Fauces und Larynx schreiten; ist man dann so glücklich, einige Hustenstösse hervorzurufen, so kann man bisweilen den Patienten aus der augenblicklichen Gefahr herausreissen.)

Als Folgezustand ist die Anämie bei wiederholten grösseren oder anhaltenden Blutungen auch in Betracht zu ziehen. Während der Behandlung ist es von hauptsächlicher Bedeutung, den Patienten psychisch und physisch zur Ruhe zu bringen, indem ihm gleichzeitig verständlich gemacht wird, wie er auf die behutsamste Weise, am besten ohne stärkeren Hustenstoss, Blut und Schleim, die heraus miissen, heraufzubefördern hat. Subkutane Morphininjektionen, die Schlaf hervorrufen dder in zu hohem Grade die Expektorationen hehindern, müssen absolut vermieden werden ${ }^{1}$ ).

Vagegen wird man sich häufig genötigt sehen, den Husten einigermassen zu beschränken - wo dieser nicht genügend beherrscht wird durch wiederholte kleinere oder mittelgrosse Dosen Kodein, Heroin oder Morphin per os. Bei grösseren oder beharrlich wiederkehrenden kleinen Blutungen scheint es vorteilhaft einen - etwas grösseren Eisbeutel anzuwenden, bis jede Spur von frischem Blute verschwunden ist, gern etwas lä̉nger. Derselbe wirkt psychisch beruhigend, vermag den Patienten in Ruhe zu halten und kann ihm die Bedeutung als ein koagulationsförderndes Moment nicht abgesprochen werden (durch Einwirken auf die Vasomotoren mit darauffolgender Hyperleukozytose. - Einspritzungen eines artfremden Serums (Diphtherieserum) tun ab und zu unzweifelhaften Nutzen, aber die Wirkung ist nicht so konstant, wie man wünschen könnte; die Rücksicht auf einen möglichen anaphylaktischen Anfall kann auch hindernd in den Weg treten. Schnelles Abbinden hoch oben an beiden unteren Extremitäten wirkt

1) Aus diesem Grunde wäre man berechtigt, subkutane Morphininjektionen als eine der grösseren Gefahren bei der Hämoptyse anzuführen. 
bei stärkeren Blutungen oft überraschend schnell und prompt. Die Binden brauchen gewöhnlich nicht viele Stunden lang anzuliegen; sie sollten ganz allmählich entfernt werden. Das Abbinden der obereu Extremitäten ist meistens nicht notwendig; vor der Entfernung der Binde von einem der Beine kann es bisweilen zweckmässig sein, einen der Arme abzubinden, um den Übergang mehr gradweise gescheben zu lassen. Die Wirkung ist früher als beruhend auf Ânsammlung einen 'Teils der Blutmassen in den abgebundenen Extremitäten erklärt worden. wodurch der kleine Kreislauf entlastet wird. Nun meint man die Wirkung besser erklären zu können als Folge einer Veränderung in dem osmotischen Verhältnis des Organismus mit einer vorübergehenden Hydrämie ausserhalb der abgebundenen Partien, hervorgerufen durch das Aufsaugen der Feuchtigkeit von den Geweben; hierdurch treten sodann Thrombokinase aus dem Gewebsparenchym in das Blut über und brechen das labile Gleichgewicht zwischen dem 'Thrombin und Antithrombin des Blutes, wodurch wiederum die Koagulationsfähigkeit des Blutes vermehrt wird ${ }^{1}$ ).

Wahrscheinlich dürften beide Momente zusammen wirken. Wo nur die eine Lunge angegriffen ist und andere Mittel fehlschlagen, dürfte ein Versuch mit dem künstlichen Pneumothorax empfohlen werden. Hier kommt dann in Betracht, dass es wahrscheinlich nur in $2 / 3$ aller Fälle glückt, eine hinlänglich freie Pleuraspalte $z u$ finden zum Anlegen des Pneumothorax; nächstdem können auch starke Verlötungen die notwendige Kompression verhindern.

Ein Mittel zum Stillen der Lungenblutung, welches das letzte Jahr hier im Sanatorium sozusagen die anderen Methoden überflüssig gemacht hat, ist die von Alexander zuerst benutzte und ron W e is mayr empfohlene Injektion grosser Dosen von Oleum camphoratum. Von 22 Patienten mit Hämoptyse im Laufe des Jahres haben 13 Fälle nur gewöhnlich beruhigende Behandlung erfordert. Bei den übrigen 9 mit mittelgrossen und grösseren Hämoptysen (40-400 g) hörte die Blutung schnell und prompt auf nach der Injektion von $3 \mathrm{ccm}$ von $20 \%$ Ol. camphor. Nur einige Male ist in unbedentender Menge frisches Blut 1-2 Tage später gekommen. Der Eisbeutel ist nicht angewendet worden, und auch keine andere Behandlung ausser der ganz gewöhnlichen ${ }^{2}$ ). - Auch bei einem Falle von hartnäckiger Epistaxis zeigte das Mittel seine Wirkung - ohne gleichzeitige Tamponade. Hier kam etwas Blut ca. 2 und 5 Wochen später.

1) Nach Morawitzs, Fulds und Speros Koagulationstheorie, die unter anderem von $\mathrm{Ham}$ marsten als die zur Zeit annehmbarste bezeichnet wird (Phys. Chemie 1914).

2) Im Laufe des letzten Monats sind bei zwei Patienten abundante Blutungen aufgetreten, welche sich einige Male mit 1-2 T'agen Zwischenraum wiederholten. Hier wurden sowohl Injektionen von Ol. camphor. als Abbinden der unteren Extremitäten angewandt, wonach die Blatung jedesmal sofort stand. Der Blutverlust geschah im Laufe der Minuten, welche hingingen, ohe die Behandlung ins Werk gesetzt werden konnte. Es muss hier angenommen werden, dass dio Blutungen durch Bersten einer etwas grösseren Arterie verursacht worden wuren (bei jedem insgesamt 2 Liter). 
Das Mittel wurde hier ganz zufällig auf Grundlage einer anderen Indikation angewandt, nämlich bei drohender Kollapserscheinungen währond des Versuchs der Einleitung von Pneumothoraxbehandlung bei einem Patienten mit hartnäckigen Blutungen. Man nahm $\mathrm{Ab}$ stand von weiteren Versuchen eine freie Pleuraspalte $\mathrm{zu}$ finden; die Kollapserscheinungen verschwanden und die Blutung hörte auf, ohne sich in dem darauffolgenden halben Jahre während des Aufenthaltes des Patienten im Sanatorinm zu wiederholen.

Ohne vorstehend erwähnten Zufall würde die - bei einem so kräftigen Herzstimulanus - supponierte Blutdruckerhöhung davor abgeschreckt haben, den Versuch mit dem Mittel zu machen, trotz der von verschiedenen Seiten geäusserten Bemerkungen betreffs der guten Wirkung desselben. Es scheint ganz unzweifelhaft, dass es sehr oft guten Nutzen tut. Es ist Tatsache, wenn es auch nach früheren Anschauungen (Blutung hervorgerufen durch erhöhten Blutdruck!) paradox erscheinen kann: Nicht einmal bei den vorher erwähnten 11 Patienten mit mittelgrossen bis zu abundanten Hämoptysen traten stärkere Blutungen nach der Injektion auf, sondern die Blutung hörte jedesmal nach Verlauf von einigen Minuten auf ${ }^{1}$ ). Indem diese einfache Tatsache referiert wird, soll nicht unterlassen werden, auch die sehr notwendige Einschränkung zu machen, dass man auch hier auf Enttäuschungen vorbereitet sein muss. - Wie bei Diphtherieserum-Injektionen kommen auch hier oft vorübergehende Temperatursteigerungen bis $\mathrm{zu} 39-40^{\circ}$ vor, was ich als Folge davon, dass eine kleine Dose Autotuberkulin in den Kreislauf hinübergespü̈lt worden ist infolge der auf die Injektionen folgenden temporären Änderungen im Blutdruck oder in der phagozytären Wirksamkeit, aufgefasst habe. Bei einer etwas grösseren Blutung kann ausserdem Änderung in dem osmotischen Druck auftreten mit Übergang von Flüssigkeit in die Blutbahn vom Gewebsparenchym, wodurch eine kleine Dose mit gespült werden kann. Auch andere Faktoren können in Betracht kommen. Für die Wirkung des Mittels können mancherlei Verhältnisse in Betracht kommen. - Die Prozedur an sich, als Injektion eines chemisch nicht ganz indifferenten - Hüssigen - Corpus alienum, muss als ein chemisch-mechanischer Reiz aufgefasst werden, weicher Hyperleukozytose hervorruft sowie reichlichen Übergang von Thrombokinasen in die Plasma des Blutes mit bedeutender Steigerung des Koagulationsvermögens des Blutes. - In Fällen, wo man die Blutungen als Folge von ve nö s er Stas e erklären kann - welcher Gedanke nahe liegt bei den vielen Phthisikern mit schwachem atrophischen Herzen -, kann die Wirkung direkt abgeleitet werden aus der gebesserten Herztätigkeit. - Aber auch in anderen Fällen scheint es natürlich, anqunehmen, dass durch eine gebesserte Herzaktion eine Entlastung des kleinen Kreislaufes vor sich geht. Macht man z. B. das Gedankenexperiment - rein schematisch - , die Arbeit der rechten und der linken Herzhälfte als $1: 3$ zu setzen, will man durch Steigerung sagen wir der Einfachheit halber: bis zur doppelten - das Verhältnis

1) Man hat vollständig den Eindruck, dass :es wie eine Digitalpression auf die blutende Stelle wirkt. 
2:6 erhalten. Im ersten Fall erhält man eine Arbeitsdifferenz von 2, im letzten Fall von 4, während die geometrische Proportion $1: 3$ und 2:6 in beiden Fällen dieselbe ist. Die Folge hiervon wird einfach die sein, dass der Druck durch 0st. arterios. sin. mehr gesteigert wird als durch Ost. arterios. dextr.; das Einsaugen (resp. der negative Druck) durch Ost. ven. sin. wird schneller zunehmen als durch Ost. ven. dextr. Aus diesen zwei zusammenwirkenden Momenten scheint man mit Sicherheit die Folgerung ableiten zu können, dass in dem grossen Kreislauf in Organen Hyperämie mit gesteigertem Turgor auftreten wird, während in dem kleinen Kreislauf eine relative Anämie mit abnehmendem Turgor des Lungenparenchyms auftritt. Wenn man die Arbeitsdifferenz der beiden Herzhälften vor und nach der Injektion bedeutend niedriger als 2 setzt, z. B. nur $\mathrm{zu}^{1 / 4}$, wird man dennoch das entlastende Moment behalten, welches die günstige Wirkung der Kampfer- und Digalen-Injektionen erklären kann, nicht allein bei der Hämoptyse, sondern - ohne sonstigen Vergleich auch bei akutem Lungenödem und Pneumonia crouposa 1). Bei demselben Quantum des Blutes im Organismus muss angenommen werden, dass die Hyperämie des Parenchyms des grossen Kreislaufes kompensiert werden muss durch eine gewisse Anämie im Parenchym des kleinen Kreislaufes. Ob man nun den Lungenkreislauf entlastet mit Hilfe von venöser Stauungshyperämie - Abbinden der Extremitäten - oder durch arterielle Hyperämie im grossen Kreislauf (wie durch Kampfer- und Digalen-Injektionen), scheint bei der Hämoptyse zu demselben Resultat zu fübren: Verminderte Blutmenge in den Lungengefässen mit herabgesetztem Blutdruck. Durch die Kombination der beiden Methoden kann die Wirkung bedeutend gesteigert werden indem die in den abgebundenen Extremitäten aufgestauten Blutmagazine weiter zunehmen werden an Volumen - , sowie auch die Gefässe und das Parenchym des grossen Kreislaufes im übrigen mehr Blut enthalten werden als unter normalen Verhältnissen. - Sowohl das entlastende, als auch das koagulationsbefördernde Moment sind bei beiden Methoden stark ausgesprochen. Und die praktischen Resultate der Behandlung scheinen in guter ubereinstimmung mit der theoretischen Grundlage der Methoden.

Auch im letzt verflossenen Jahre - seitdem das Vorangehende geschrieben ist - sind wir bei der Behandlung von Lungenblutungen mit Hilfe der zwei genannten Mittel zum Ziel gelangt, ohne zu diesem Zweck den Pneumothorax oder Eisbeutel anwenden zu müssen. Nur bei einem einzigen Fall standen wir bei einer kopiösen Lungenblutung, im Begriff, den Pneumothorax anzulegen; aber die Blutung hörte auf nach $3 \times 3 \mathrm{ccm}$ Ol. camphor. und dem Abbinden der Unterextremitäten im Laufe eines Tages. - Durch die stark gesteigerte Wirksamkeit der linken Herzhälfte als Drucksaugpumpe kann vielleicht die Möglichkeit eines gesteigerten Risikos.- Embolus zu

1) Bei einem Patienten mit komplizierender Schrumpfniere und Herzhypertrophie traten - mit einer Pause von einer Woche - ein paar Anfälle von akutem Lungenödem mit profuser Sekretion einer sero-sanguinolenten Flüssigkeit sowie Suffokationserscheinungen auf. Die Anfälle verschwanden schnell nach der Injektion von $3 \mathrm{ccm}$ Ol. camphor. $(20 \%)+1 \mathrm{ccm}$ Digalen. 
dem grossen Kreislauf von einer arrodierten Lungenvene oder einer grösseren Wundfläche auszutreiben ${ }^{1}$ ) - nicht ganz ausgeschlossen werden. Aber die prompte hämostatische Wirkung der Behandlung ergibt - nach dem bisherigen Anschein - unserem Dafürhalten nach so grosse Vorteile für die meisten der Patienten, scheint die Patienten vor so vieler Gefahr anderer Art zu behüten, dass wir nun, nachdem wir die Behandlung über zwei Jahren angewendet haben, uns dieselbe durch andere Mittel kaum ersetzt denken können.

1) Vgl. Dunins Fall, ref. in Begtrup-Hansens Doktor-Dissertation: Über die Pneumothoraxbehandlung. 